\title{
In situ EELS Study of the Thermochromic Transition in $\mathrm{VO}_{2}$
}

\author{
M.T. Ochoa-Lara, H.E. Esparza-Ponce and F. Espinosa-Magaña
}

Centro de Investigación en Materiales Avanzados, Laboratorio Nacional de Nanotecnología, Miguel de Cervantes No.120, C.P. 31109, Chihuahua, Chih., México.

Recently smart materials have become very important in applications [1-2], like transducers, actuators, etc. Thermochromic materials are temperature sensitive products used for smart windows coating. $\mathrm{VO}_{2}$ is a classical thermochromic material that undergoes a transition at $67^{\circ} \mathrm{C}$ [3-4] from high temperature metallic phase (tetragonal structure, rutile type) to a lowtemperature insulator phase (monoclinic structure), has been considered as an intelligent window coating which can to control solar energy transmission.

The goal of this work is the in situ to study of this metal-insulator transformation by EELS to have a deeper insight about the electronic structure and identifying both tetragonal and monoclinic phases. The identification of the phases is supported by XRD figure 1 and DSC studies figure 2 . We found useful and interesting to carry out a study in polycrystals because it is easier to manipulate this material, is cheaper than single crystal structures, whose fabrication is more expensive.

The phase transition temperature was found at $69^{\circ} \mathrm{C}$, determined by differential scanning calorimetry (DSC) and at the minimum of this endothermic peak is associated with the first modifications of the absorption bands. XRD studies support this result, showing shifting of the characteristic peaks after transformation has taken place above $70^{\circ} \mathrm{C}$.

The thermochromic transition was induced by placing the sample in a TEM heating sample holder and EELS spectra were acquired at room temperature and $120^{\circ} \mathrm{C}$, corresponding to monoclinic and tetragonal phases, respectively. In this way, we generated an in-situ heating process that allowed us to obtain spectra for monoclinic and tetragonal structures from the same region in the sample. Electron energy loss spectra were obtained using a Gatan Parallel Electron Energy Loss Spectrometer (PEELS model 766) attached to a transmission electron microscope (TEM, CM200). Data were acquired in diffraction mode with $0.2 \mathrm{eV} / \mathrm{ch}$ dispersion, an aperture of $2 \mathrm{~mm}$ and a collection semi-angle of about $2.7 \mathrm{mrad}$. The resolution of the spectra was determined by measuring the full width at half-maximum (FWHM) of the zero loss peak and this was typically close to $1.1 \mathrm{eV}$, when the TEM was operated at $200 \mathrm{kV}$. EELS spectra were corrected for dark current and readout noise. The channel to channel gain variation was minimized by normalizing the experimental spectrum with independently obtained gain spectrum of the spectrometer. Next, all spectra were deconvoluted by the zeroloss peak (ZLP) recorded in a hole of the grid to obtain single scattering distributions $\mathrm{S}(\mathrm{E})$. The real and imaginary parts of the dielectric function can be obtained from the energy loss function $\operatorname{Im}(-1 / \varepsilon)$, through Kramers-Kronig analysis. Fig. 3 shows the energy loss function of $\mathrm{VO}_{2}$, before and after the thermochromic transition. It is observed a small shift in plasmon peak position, towards higher energies for tetragonal phase.

\section{References}

[1]J Frederic Guinnetona, Laurent Sauques, et.al, J.of Physics and Chemistry of Solids 66 (2005) 63-73.

[2]E.Z. Kurmaev, V.M. Cherkashenko, Yu M. Yarmoshenko, St. Bartkowski, A.V. Postinikov, M.Neumann, L-C.Duda, J.H.Guo, J.Nordgren, V.A. Perelyaev, and W. Reichelt, J. Phys. Condens. Matter 10, 4081,(1998).

[3]. F. J. Morin, Phys. Rev. Lett. 3, 34 (1959)

[4]. R.M. Wentzcovitch, W.W. Schulz, and P.B. Allen, Phys. Rev. Lett. 72, 3389 (1994) 


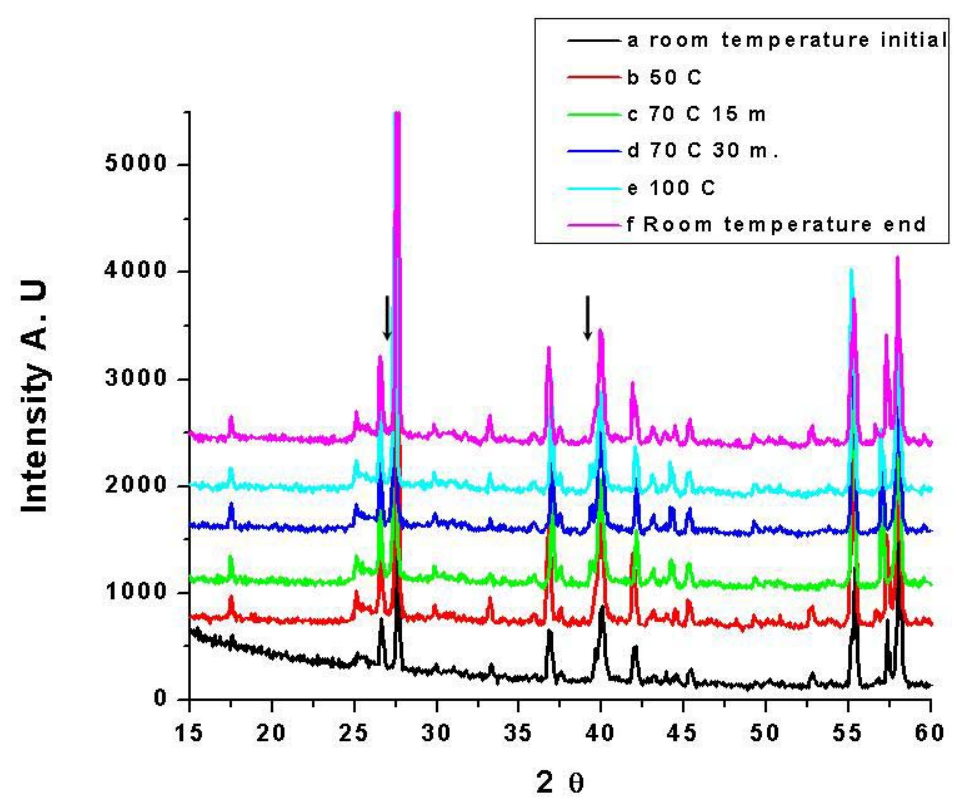

Fig 1. XRD from $\mathrm{VO}_{2}$ showing characteristic peaks at (a) room temperature, (b) $50^{\circ} \mathrm{C},(\mathrm{c}) 70^{\circ} \mathrm{C}$ after heating for 15 minutes, (d) $70^{\circ} \mathrm{C}$ after heating for 30 minutes, (e) $100^{\circ} \mathrm{C}$ and (f) room temperature end

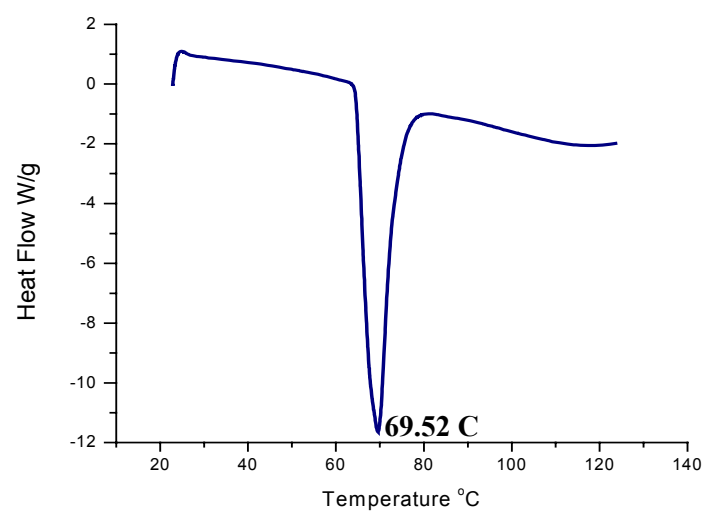

Fig. 2 DSC analysis of $\mathrm{VO}_{2}$ powders heated under argon atmosphere (heating rate $5^{\circ} \mathrm{C}$ $\min ^{-1}$

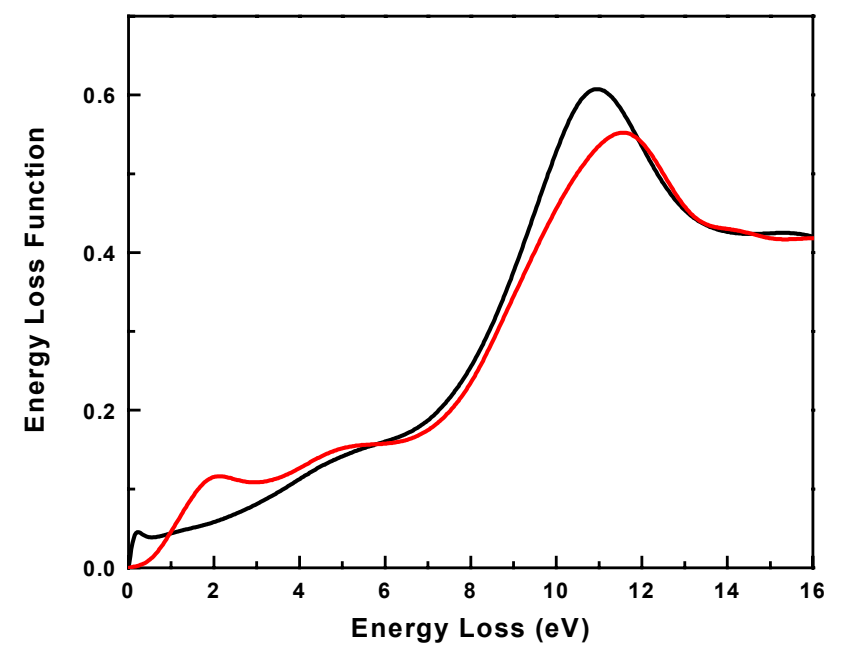

Fig. 3. Energy loss function of $\mathrm{VO}_{2}$ for monoclinic (black) and tetragonal (red) structures. 